\title{
Cosmic metaphysics: Being versus Becoming in cosmology and astrophysics
}

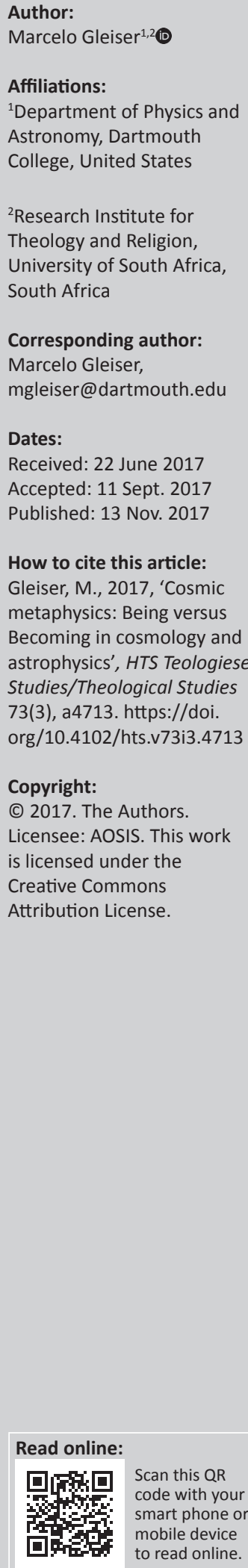

This article offers a critical discussion on the question of the existence of the Universe, starting with creation myths of a variety of different cultures and ending with cutting-edge ideas from modern cosmology. Emphasising the polarised tension between Being and Becoming and its religious and philosophical origins, this article argues that this tension is unavoidable and still very much present. Further, the logical insolvability of the problem of the First Cause is examined, showing the conceptual inappropriateness of current scientific models that claim to offer a solution.

\section{Which metaphysics?}

Given that this article will be discussing the role of metaphysics in cosmological and astrophysical thought, we should start by defining what is meant by metaphysics, a word that incites adverse reactions from most scientists and even philosophers, in particular, logical positivists. According to the Oxford English Dictionary, metaphysics is:

the branch of philosophy that deals with the first principles of things or reality, including questions about being, substance, time and space, causation, change, and identity (which are presupposed in the special sciences but do not belong to any one of them); theoretical philosophy as the ultimate science of being and knowing. (OED: http:/ / www.oed.com/view/Entry/117355?redirectedFrom=metaphysics\#eid)

Following this definition, metaphysics deals with the conceptual scaffolding upon which the physical sciences are built. It is no accident that Isaac Newton, in the Scholium of his groundbreaking Principia, needed to define space, time and matter in order to formulate his laws of motion and universal gravitation (Newton 1687:408). It is also no accident that, much later, Hermann Weyl's masterful exposition of Einstein theories of relativity was titled Space, Time, Matter (Weyl 1922). Thus, for the sake of this article, we will consider metaphysics to be the branch of philosophy that deals with the fundamental aspects of physical reality, in particular, time, space, substance and change. We can call it a physical metaphysics, to distinguish it from questions arising from moral philosophy, concerned with the nature of justice and values. With this definition in mind, we will embark on a critical examination of metaphysics thinking in cosmology, starting from the very beginning, pre-scientific cosmogonical (creation) myths.

\section{Creation myths}

With very few exceptions, such as the Pirahã natives of the Brazilian Amazon forest and, in a very different sense, Buddhism, most cultures have a creation narrative, or creation myth. Such narratives address, in often beautifully poetic imagery, the question of the origin of all things: How did the world come to be? How did people and animals come to be? Taken within a broad cultural context, it's no surprise that modern-day scientists are as fascinated with the question of origins as were the shamans of our distant ancestors.

Religions across the globe and across time have dealt with the question of the origin of all things in similar ways. To create the world, with all its material things, there needs to be a stage where things exist - space - and an account of how their histories unfold - time. To create space and time and the things within them, religions invoke deities capable of existing outside the confines of space and time - and with the power to create things within space and time. So, by definition, to be a god is to transcend the confines of space and time, to exist beyond the boundaries that define our existence. The gods, or God, do not respect the laws of nature that constrain material and living things. They transcend the dichotomies that define our existence.

In my book The Dancing Universe, I argued that there are only five possible answers to the question of creation, and that all religious creationaa myths choose one of them. We may call them 


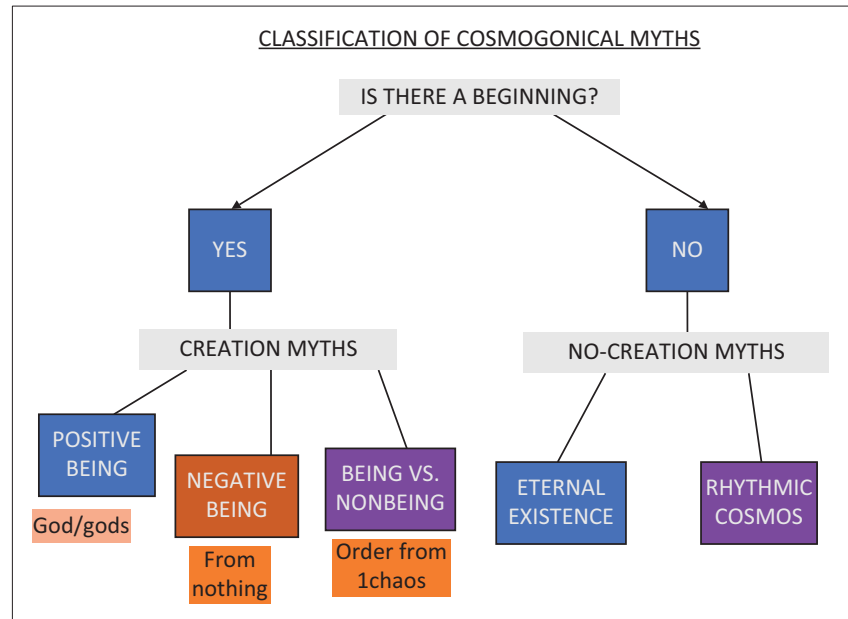

FIGURE 1: A classification of cosmogonical myths.

'archetypes of creation' (Gleiser 1997:9). First we organise the mythic narratives by whether there was a moment of creation, a moment when cosmic time started to tick. There can only be two answers to that: yes, time started at some point in the past; or, no, the universe is eternal. Within each, there are different possibilities organised as in Figure 1.

I call the 'no-beginning' myths those without a single beginning of time. Within those, there are two possibilities: an eternal cosmos, without beginning and end; and a cyclic cosmos, where the cosmos is created and destroyed in cycles that repeat throughout eternity, with no moment of creation more important than any other. The Jains of India espoused the eternal cosmos, while the Hindus espoused cycles of creation beautifully represented by the dance of Shiva.

Myths with a beginning, the most common by far (including the Old Testament), invoke a God or gods who create the world at a specific moment in the past. There are, however, also myths with a beginning where there is no deity involved, as in the Maori creation narrative 'out of nothing'. Barbara Sproul's book Primal Myths offers a wonderful collection of creation myths (Sproul 1979). Finally, order may emerge out of chaos, with or without divine intervention.

This classification illustrates the tension between Being and Becoming within pre-scientific narratives of Creation. All take Being as primary and timeless, with Becoming as the descriptive flow of material transformations. The primacy of Being is clear in the assumption of an Absolute power, the creative deity that transcends the boundaries of space and time. As we move to pre-Socratic Greece, we will identify a similar tension in the philosophies of the Ionian and the Parmenidian schools and their followers.

\section{Being versus Becoming in ancient Greece}

From Thales to Democritus, pre-Socratic philosophers were deeply engaged in unpacking the metaphysical foundations of reality (Kirk, Raven \& Schofield 1983). The tension between the two polar extremes, Being and Becoming, is apparent in their fragmentary texts and responses to one another. That Zeno, a disciple of Parmenides of Elea, would propose his famous paradoxes, such as Achilles and the Tortoise, to deny the importance of the senses, is a powerful illustration of how high the stakes were.

Thales and his Ionian successors proposed, right at the inception of Western philosophy around $600 \mathrm{bce}$, a unified theory of Nature whereby all stuffs of the world were but different manifestations of a primal substance, the embodiment of a reality always in flux. To the Ionians, time was the essence of reality. In contrast, Parmenides and his followers proposed that the essence of Nature was not to be found in the transient but in the permanent; that what is cannot change, for it then becomes what it is not. The truth, they argued, could not be ephemeral. To them, timelessness was the essence of reality. In the relatively short time span of 100 years, philosophies of Being and of Becoming were proposed as mutually exclusive pathways to Nature's secrets.

Thales and the Ionians argued for a unified ontology, a primal substance that gives rise to all that is. They were proposing a unified theory of Nature, based on specific materials as the primal source of existence. That Thales chose water, Anaximenes air and Heraclitus fire is less important than their ontological focus on a single substance capable of transforming itself, representing the ephemeral nature of physical processes. AS Heraclitus wrote in Fragment DK B10: 'The one is made of all things, and all things issue from the one' (Heraclitus Fragments: DK B10). Nature is always in flux, as beautifully captured in Heraclitus' famous phrase, 'On those who enter the same river, ever different waters flow' (Heraclitus Fragments: DK B13). Theirs was a metaphysics of Becoming.

In contrast, Parmenides and the Eleatics saw the essence of reality as unchanging, what they called to eon, What Is, or alêtheia, True Reality. They proposed that change is an illusion, a distortion our imperfect senses cause in how we perceive reality. These are some of the earliest considerations in the West about the nature of reality and our perception of it: Is the essence of reality 'in your face', the transformations we witness with our senses? Or is its essence hidden, locked in an abstract realm perceived through thought alone?

To perceive change, we need to sense it. But if our senses feed us only imperfect reconstructions of what exists, how can we grasp what is truly real? On the other hand, if we follow Parmenides, how can we possibly have any idea of this 'thing' that doesn't change? After all, if something doesn't change, it becomes imperceptible to us, like a humming to which we grow deaf. Worse, if this unchangeable reality exists somehow in a rarefied realm, how are we to make sense of it? How can we probe it? And so, the Ionians would accuse the Eleatics of empty abstractions, while the Eleatics would think the Ionians fools, as they trusted what could not be trusted. Meanwhile, the Pythagoreans would ignore both, following their belief in the power of mathematics to describe 
the harmony and beauty of the world. To them, number was the essential ontological substrate upon which all reality was based.

The stage was thus set for Plato, who sided with Parmenides while incorporating essential aspects of the Pythagoreans: the essence of reality cannot change and it is captured through mathematics, in particular, through geometry. Reality reduced to shapes, the perfect platonic solids, arranged in multiple ways, coexisting with Ideal Forms - the archetypes of potential existence. Once they 'materialise' and become part of sensorial reality; they lose their perfect attributes, joining the imperfections of the world. To rely on the senses, as Plato makes clear in his Allegory of the Cave (Plato: The Republic) is a sure path to error and delusion. Only in reason, within Mind, can we grasp the true essence of reality. Plato clearly sided with Being.

Aristotle responded in the opposite direction, bringing down philosophy from the rarefied realm of pure reason to the dirty reality of the world, with its objects and incessant change. However, a philosophy purely based on terrestrial transformations would not be able to describe the heavens and its (apparently) fixed patterns. As a solution, in his cosmology, Aristotle separated the cosmos into two separate realms, sub-lunar and supra-lunar, with the Moon demarcating the boundary between Becoming and Being. Below the Moon, the four basic elements (water, earth, air, fire) would combine and separate, promoting the changes and transformations we witness. In contrast, the Moon and all other celestial luminaries were unchanging, made of a fifth essence or quintessence, the eternal ether. If some claimed the Moon appeared imperfect to the eye, Aristotle and his followers would counter, arguing that it is but an illusion from the rising fires from below. Meteors (in particular meteorites) and comets were described as sublunary, or atmospheric phenomena. Indeed, the word 'meteorology' is still used to describe the weather, an inheritance from Aristotelian thought.

With this division of the cosmos into two non-overlapping realms, one of Becoming and the other of Being, Aristotle sought to reconcile the two warring metaphysics of his antecessors. Motion was imparted into the cosmos from outside in, from the outermost sphere of his onion-like cosmos, through the action of the Unmoved Mover, without which 'there will be no first principle, no order, no becoming, no heavenly bodies ...' (Aristotle, Metaphysics).

Aristotle thus solved the problem of the First Cause similarly to creation myths, based on an Absolute being, a power without material extension or duration, capable of imparting motion onto other unmoved movers, in a hierarchy that descended all the way down to ordinary material things. In order to do so, the Unmoved Mover had to act through intellect, the perfect good, because that which is not material couldn't physically impart motion into what is. We find such ideas resurrected in current cosmology, where adherents of the strong Anthropic Principle presuppose a cosmic teleology that ultimately engenders life (Barrow \& Tipler 1988).

The Greeks had thus formulated the essential components of cosmic metaphysics as divided into two main camps: Being and Becoming. Parmenides and Plato defended that Truth can only be reached through the world of thought, that the senses, the transformations we see in the world around us and within us, are illusory and deceitful. Inspired by Plato, later Neoplatonists combined his ideas with that of the Pythagoreans emphasising that truth must be expressible through mathematics, and that mathematical symmetry is equated with beauty, effectively creating a rational aesthetics of Nature. The philosopher would thus decipher Nature's blueprint through the diligent application of mathematics and the observation of the heavenly motions. For the great Claudius Ptolemy, as for Plato and Aristotle, the heavenly bodies were divine, and the order perceived in the Universe was a manifestation of superior Reason. The study of the heavens would lift the astronomer from the crudeness of everyday life into the realm of the gods, pointing the way to a higher moral and ethical existence. By investigating the workings of the cosmos, the astronomer would be in touch with the divine.

On the other hand, the Ionian school believed in a world in flux, always in transformation. A curious exception is Anaximander, a true visionary, who proposed a primal essence from whence everything emanates and goes back to, the Boundless. In doing so, he is the first to attempt a reconciliation of Being and Becoming, at least in the West. After him, the Atomists Leucippus and Democritus also attempted a reconciliation, this time proposing immutable atoms (Being) that nevertheless could combine into myriad forms, explaining the changes we see in the world (Becoming).

As we move into the Renaissance, such notions would greatly inspire and influence the patriarchs of modern astronomy and science: the search for immutable truths about the world, expressible through mathematics; the search for the fundamental stuff of reality; descriptions of material transformations and of the apparent order in the heavens; the role of the divine in guiding and designing natural phenomena. Such are the metaphysical questions and challenges that will dominate the works of Copernicus, Galileo, Kepler, Descartes and Newton and that will drive their science.

\section{Being and Becoming at the cradle of modern science}

We do not need to provide an overview of the great transformations in human thought that occurred during the 17 th century, as the topic has been greatly explored elsewhere (Gleiser 1997; Koyré 1973; Westfall 1983). For us, the essential point is that from Copernicus to Newton, the new scientific cosmology was by necessity, Being-based. I mean by necessity because of the fundamental role that religion played as the 
conceptual scaffolding for all of these men. What is remarkable, and that we will explore further, is how Becoming sneaks in as if from the back door, very much against the will of these natural philosophers.

Copernicus was a canon of the Catholic Church and dedicated his On the Revolutions of the Heavenly Spheres to Pope Paul III. The new cosmic aesthetics he proposed was in tandem with the Greco-Roman revival of the Renaissance, using an ordering proportion based on the planetary orbital times around the Sun. Indeed, as he claims in his dedication letter to the Pope:

I began to be annoyed that the movements of the world machine, created for our sake by the best and most systematic Artisan of all, were not understood with greater certainty by the philosophers ... (Copernicus 1543)

The cosmos was created by God and, as such, needed to reflect His artisanal skills. Unfortunately, when he tried to use Ptolemy's old data to fit his heliocentric scheme, Copernicus realised something was amiss. (It was the shape of the orbits, which he insisted should be circular.) In an attempt to 'save the phenomena', to use Plato's phrasing, Copernicus used not only epicycles but also epicyclets, smaller epicycles nested within larger ones: clearly, not a highly perfected work for a divine and all-powerful Cosmic Artisan.

Even heretic Galileo, one could argue, was motivated by his devotion to the Church and not by a well-thought campaign to undermine its authority, as he certainly ended up doing. Knowing that an Earth-centred cosmology was untenable given what he had been discovering about the skies, Galileo wanted to save the Church from future embarrassment. Granted, his style was not the most diplomatic. However, neither were the Inquisition's and its crushing blows to ideas contrary to Christian theology. Galileo also got marred by circular orbits, and had to revert to an Aristotelian notion of 'circular inertia' to justify the orbits of the heavenly luminaries. He identified sunspots and craters and mountains on the Moon, contrary to the perfect heavenly order of Aristotelian cosmology.

In Kepler, we find a true Pythagorean, a revolutionary thinker with one foot firmly planted in the past and another in the future. Armed with Tycho Brahe's highly precise astronomical data, Kepler attempted to fit planetary motions into circular orbits. In the end, he famously had to adopt ellipses as the shapes that best fit the data, a truly modern outlook. Asymmetry had crept into the cosmic architecture, signalling the breakdown of the perfect Aristotelian cosmos, failing to reflect in its motions the symmetric purity of a divine aesthetic.

The picture becomes even more alarming when we add Brahe's discoveries that further undermined the Aristotelian framework of non-overlapping domains of Being and Becoming. With the New Star of 1572 and the Great Comet of 1577, Brahe showed that change was indeed possible above the lunar orb, sabotaging the long-held notion that there couldn't be any change in the heavens. 'Becoming' was now extended to the skies, bringing them closer to the imperfect and always changing realities of men.

Newton will finally offer a conceptual and mathematical framework to unify the Earth and heaven, proving the universality of gravity: the same force that makes the apple fall also is responsible for the orbit of the Moon around Earth and of the Earth around the Sun. Extrapolating outwards, Newton would speculate that the attractive properties of gravity, when applied to the distant stars, imply that the cosmos itself cannot be shaped as a finite sphere but must, instead, be infinite. Otherwise, as he argued in a response letter to Rev. Richard Bentley, all worlds would collapse into one central point: gravity made the cosmos unstable. Instead, God, in His mysterious ways, provides equilibrium to the infinitely many attractive pulls in opposing directions that stars exert on each other, an unstable cosmic equilibrium not unlike balancing pins on their ends. Newton's was a theistic cosmos, where God played an active dynamic role. Again, a cosmos nourished by Being couldn't escape the intrusion of change and transformation in its very essence. Like it or not, asymmetries and instabilities seemed to play an essential role in the cosmic dynamics. Becoming refused to play second fiddle.

It is thus clear that even as modern science starts to take shape during the 17th century, the hoped-for domination of a metaphysics of Being - inspired by a monotheistic tradition transposed to the nature of the cosmos - is progressively tainted by the dynamics of natural processes in general, which clearly demonstrate some of the precepts of a metaphysics of Becoming: widespread change, instabilities, asymmetries, flow and transformation. Still, the 18th century will see a very hard push towards an even more complete rationalisation of Nature, with an increasing emphasis on natural law and the mechanisation of physical reality. The allure of Being was hard to resist.

\section{Natural laws and the clockwork cosmos}

The incredible predictive and descriptive success of Newton's mechanics and theory of universal gravitation drove physics and astronomy into a new phase whereby the cosmos and its motions became comparable to that of a clockwork: precise and precisely determined through mathematical laws that combined diverse phenomena under a single umbrella. For example, a single theory of gravity described the tides, the orbits of comets and the Earth's oblate shape. The notion that the physical properties of the Universe were describable through a strict mathematical formulation took root, and physics became synonymous with the search for the laws of Nature, the set of mathematical statements that best synthesised Nature's blueprint. Curiously, and to what would amount to Newton's discontent would he witness such transformation, the advancement of science rescued the old Aristotelian idea of the Unmoved Mover, as God became 
identified with the designer of the cosmos and of its mathematical laws. Once created, the cosmos did not need the interference of a meddling deity: the cosmos of the Theists gave way to the cosmos of the Deists, as God's interference in the affairs of the world was relegated to that of the cosmic clockmaker.

As the notion of Being was apparently being pushed to the very beginning of time, and thus possibly fading into oblivion, a reacting trend came to dominate the minds of 18th-century natural philosophers, who tried to rescue the Platonic-Pythagorean ideal. While it is true that particles of matter are pushed about by forces, the motions that are generated satisfy conservation laws that, in themselves, are unchangeable. We see here how Being is rescued into the very heart of the physical sciences, as a mathematical blueprint expressed through unchangeable laws of Nature. Clearly, understanding such laws was akin to 'knowing the mind of God', an image that remains strong today among certain theoretical physicists.

First was Lavoisier, with his law of mass conservation around 1785: in a chemical reaction matter is neither created nor destroyed. So, although chemistry is the science of material transformations, underneath the change we detect the unchangeable, the quantity of matter itself. Time-independent principles underlie the material changes and transformations that the physical sciences describe.

This conceptual framework became solidified once JosephLouis Lagrange pioneered the calculus of variations and the Lagrangian formulation of classical mechanics, which led to the famous Euler-Lagrange equations of motion. Lagrange's formulation was later refined and extended by William Rowan Hamilton. The goal of mechanics is to obtain the exact paths in space that the particles of matter will undertake when acted by forces. Every physical system is characterised by a quantity known as its Lagrangian, which, in Hamilton's formulation, can be written as the difference between its kinetic and potential energies (Lanczos 1970). The integral of this quantity between two times, the initial and final times of motion, is called the 'action'. Perturbing the action between these two fixed points in time and stating that such perturbations minimise the action generates the equations of motion for the system, the Euler-Lagrange equations. Remarkably, Nature chooses the motion corresponding to the path that minimises the action between the initial and final times: Becoming (the motions of material particles) is generated from perturbing Being (the action).

In principle, every mechanical system comprised of particles has an action, and the minimisation of this action leads to the equations of motion describing the paths the particles undertake under the action of various forces. The motions could be of planets around the Sun, or of billiard balls on a pool table. This principle of least action plays an essential role in the mathematical formulation of mechanical laws, and offered tremendous impetus to carry the Enlightenment programme even further. In the early 1800s, Pierre Simon de
Laplace offered a robust analysis of the dynamics of the solar system in his impressive Celestial Mechanics, which included a mathematical model for the formation of the solar system based on the law of conservation of angular momentum. Again, a mathematical law of Nature based on an unchangeable (conserved) quantity provided the foundation to explain the transformation from a primordial nebula to a solar system with a central star and orbiting planets.

Laplace's famous Supermind, a revival of Plato's Demiurge, illustrates the inflated confidence in this strict cosmic determinism. If it could know the positions and velocities of all the particles in the Universe at the same time, the Supermind could predict every future motion. This being the case, and given that the mechanistic view held that everything is made of particles moving under the action of forces, the Supermind could predict everything: when comets appear, when you were born, when you'd marry, die, etc. In such a cosmos, there would be no room for free will, given that everything would be controlled and known by the Supermind. Even if only an allegory, the Supermind betrays the spirit of the times. It is no wonder that the Romantics would angrily react to such an imprisonment of the human spirit.

Thankfully, the Supermind is a physical impossibility because for it to know the positions and velocities of all existing particles at the same time it would have to be omnipresent and omniscient, something that clearly violates causality, at least for a system that satisfies natural laws. By necessity, Laplace's Supermind would have to be supernatural.

In his spectacular mathematical formulation of celestial mechanics, Laplace left open the essential question of origins: where did the primal nebula that would become the solar system come from? During the 1800s, advances in astronomy, because of rapid progress on the observational range and precision of telescopes, would reveal a remarkable diversity in the heavens, to which William Herschel referred to as the cosmic garden, where nebulae of all sorts and colours were spotted along with the stars of the night sky. Clearly, dramatic physical processes generated deep change and transformation in the skies. The cosmic clockwork would have to coexist with a Heraclitean reality of Becoming, dominated by fire and renewal. Some physical systems seem to go beyond the strict deterministic dynamics of Lagrange-Hamilton, needing a different set of laws or even new physics for their description.

Soon after Celestial Mechanics, physics was to see its horizons enlarged to include electromagnetism and thermodynamics, and the need for a continuous - as opposed to a discrete or particle-like - description of physical systems. This is where the all-important concept of field entered the scene, thanks to the Michael Faraday-James Clerk Maxwell formulation of electromagnetism. A new theory of Nature based on the temporal evolution of continuous quantities generated by sources of electric and magnetic fields led to a remarkable discovery: light as a waving electromagnetic field, travelling at incommensurate high speed. 
This discovery would force physicists to consider the existence of a new medium in Nature, one that supported the propagation of light waves. After all, just as sound waves need air and water waves water, where do light waves propagate? To work as needed, such medium would need to have very bizarre properties: perfectly transparent (so as not to obfuscate the light from distant stars), imponderable (given that we do not feel its gravitational pull, nor does it offer any friction to the orbits of planets) and extremely rigid (given that it must support the propagation of waves at such fast speeds). Hence was the ether reborn, rescued from Aristotelian physics to serve the mechanistic needs of 19thcentury natural philosophers. As in Aristotle, this luminiferous ether was to fill the entirety of space, being incorruptible and immutable. It would be Being incarnate.

We see here a dual role for Being during the very active second half of the 19th century: on the one hand, we identify conservation laws and principles as the immutable mathematical blueprint upon which the varieties of natural phenomena unfold, here and everywhere in the cosmos. The Lagrangian-Hamiltonian formulation of mechanics is reinforced by the discovery in 1842 of the law of conservation of energy, giving a new dimension to Lavoisier's principle of mass conservation within the context of thermodynamics, the study of heat. The Universe obeys strict conservation laws expressed mathematically. On the other hand, with the ether hypothesis we see a possible materialisation of Being, a 'thing' that exists in the world even though it does not partake in the natural formulation of material entities. Ironically, we see echoes of the same issues reappearing with the advent of dark energy in astronomy (Gleiser 2014).

\section{The universe according to Einstein}

As is well known, in 1905 Einstein decreed the ether dead with his special theory of relativity. There was no need to propose such a strange material medium: light travelled (albeit mysteriously) in empty space. After the failure of the 1877 Michelson-Morley experiment to detect an 'ether wind', Einstein's discovery was initially welcomed only by a handful of influential physicists, such as Hendrik Lorentz, Hermann Minkowski, Henri Poincaré and Max Planck, finding resistance in others (including Michelson, who died in 1931 still believing in the ether!).

The formulation of Einstein's relativity theory is a clear illustration of the rooting of Platonist ideals in his work. Despite its name, the theory is based on two invariants, one that the laws of Nature are the same for all inertial observers (travelling with constant relative velocities), and the other that the speed of light is always constant, irrespective of observer (Einstein 1952). If we equate Being in physics with invariant principles, Einstein was clearly after those.

The phenomenal success of Einstein's special and general theories of relativity, having been validated through many terrestrial and astronomical observations, revived with tremendous impetus the Platonist effort to build a purely mathematical, all-encompassing theory of Nature. However, side by side with Einstein's theories, the emergence of quantum theories was to become a difficult adversary. Although the quantum world was rich with symmetries and conservation laws, its mixing of observer and observed seemed to mock the realists' pursuit for a 'view from nowhere' (Nagel 1986), a depersonalised, objective view of physical reality. Even the notion of existence was put into question, given that one could only affirm that an electron 'existed' once it was detected. The classical notion of a path where a particle goes from here to there became meaningless. Instead, physicists were left with a probabilistic description of physical phenomena, very much symbolising the notion of Becoming. Light becomes matter; matter becomes light. Particles exist upon being observed, having only the potentiality to exist before the act of observing (Gleiser 2014).

It is no wonder that Einstein resisted the challenging quantum reality, believing to the end of his life in an underlying order, rational and comprehensible to the human mind. He spent the last two decades of his life pursuing an extension of general relativity that would unify gravity with electromagnetism, thus expanding the realm of Being to the forces that can be measured outside the atomic nucleus. In parallel, his views of the Universe were also forced to change because of unexpected astronomical discoveries.

\section{Cosmic expansion}

In 1931, Einstein went to visit the American astronomer Edwin Hubble at the Mount Wilson Observatory outside Los Angeles. During the 1920s, Hubble had made two tremendous discoveries: one that the Milky Way was but one among hundreds of billions of other galaxies; the other that such distant galaxies were moving away from one another. This galactic receding motion was interpreted as a remarkable consequence of Einstein's general theory of relativity, the plasticity of space (and time). To accommodate the invariance principles needed to formulate the theory (same laws of Nature for all observers, constant speed of light, conservation of energy and momentum), space and time had to become stretchable, responding to variations of the concentration of matter and energy.

In 1917, soon after the original formulation of his theory, in an attempt to describe the geometry of the Universe as a whole, Einstein had proposed the first model of modern cosmology (Einstein 1952:175). He assumed, quite reasonably given the data at the time and very much consistently with his Platonic view of Nature, that the Universe was maximally symmetric (shaped as a three-dimensional sphere) and static: in effect, a finite space without a boundary, a perfect manifestation of Being, echoing Nicholas of Cusa's view of a perfectly symmetric theological cosmology. Natural phenomena took place within this perfectly symmetric space, consistently with the locality of change and transformation. According to Einstein, Being was the essence; Becoming the detail: an important detail, of course, but secondary to the framework of existence. 
His meeting with Hubble would force him to radically change his mind. The data were quite clear, and pointed to change not only at the local level of galaxies but at the Universe as a whole. Suddenly, the cosmos was endowed with a history, with a beginning and, presumably, an end.

The discovery of cosmic expansion brought the question of origins back to the forefront of science. After all, because the cosmos had a history and was expanding, looking sufficiently back in time would necessarily lead to a point of maximal compression of matter, an effective beginning of the cosmic history. In the 1960s, Stephen Hawking and Roger Penrose proved a series of singularity theorems, according to which, under very reasonable assumptions of positivity of energy, the Universe would start from a spatial singularity, where the density of matter and the curvature of space would diverge to an infinite value (Hawking \& Ellis 1973; Penrose 1965). The problem of the First Cause came back to haunt modern cosmologists.

As we discussed in section 'creation myths', creation myths of diverse cultures proposed five possible 'solutions' to the problem of the First Cause, all but one presupposing the existence of some Absolute power that transcends the boundaries of space and time (The exception is the 'creation out of nothing' modality, whereby the Universe simply came to be without divine intervention). As I noted in The Dancing Universe, after Einstein's pioneering work on modern cosmology, cosmologists developed a variety of possible solutions to his set of equations, each proposing a different kind of universe, some with a history, others not (Gleiser 1997:303). Indeed, before data were available to help discriminate between theoretical models, a plethora of 'desktop universes' appeared in the literature, motivated by personal choice and mathematical integrity, but by little physics.

Again, we can arrange the different possibilities into five classes, according to whether the universe had a specific beginning in time or not. If not, there are two choices: a cyclic universe that expands and contracts periodically, which is a possible solution of the Friedmann-Lemaître universe with a closed geometry; or a globally static universe, as in the 1948 Steady-State model of Hermann Bondi, Fred Hoyle and Thomas Gold. In this case, even if the universe does expand, space is created to fill in the vacant stretches so as to have the whole expanding effect averaged out. In order to do this, the model must violate energy conservation, an apparent contradiction. However, it does so at such minimal levels as to escape any possibility of detection.

Universes with a beginning again divide into three possibilities. A universe with a clear beginning of time, as in the big bang model; a universe which comes 'out of nothing', as in the 1973 Edward Tryon's quantum creation of the universe and its descendants proposed by several physicists, including the no-boundary initial state of Stephen Hawking and James Hartle; and a universe out of chaos, from the socalled mixmaster model of Charles Misner, further developed by V.A. Belinsky, I.M. Khalatnikov and E.M. Lifshitz in 1970.
The qualitative parallels with creation myths are clear, although, of course, scientific models do not invoke an Absolute power to deal with the First Cause. Indeed, scientific cosmological models cannot frontally address the issue. The best that can be done is to propose a quantum initial state of the universe, as was done in the Hawking-Hartle model, although it suffers from a series of technical shortcomings. Even if it did not, it would still need the whole framework of quantum physics and general relativity to be formulated, as well as a set of essential laws of Nature, in particular the conservation of energy and momentum.

The situation changed dramatically with the 1965 discovery of the cosmic microwave background radiation, a prediction by George Gamow, Ralph Alpher and Robert Herman as they developed the big bang model in a series of articles in the late 1940s and early 1950s (Alpher \& Herman 2001). With the cosmic microwave background, there was little doubt that the universe did indeed emerge from a hot and dense initial state long ago, which modern observations put at 13.8 billion years. The Steady-State model had to be abandoned, and physicists changed gears to understand the dramatic conditions that prevailed near the beginning of time, when physics as we know it breaks down.

\section{The early universe and the multiverse}

With the confirmation of the big bang model, it became clear that to understand the cosmic infancy, physicists needed to push their theories well beyond what was testable with current technology. As an illustration, the record-breaking energies achieved at the Large Hadron Collider at CERN are about 15 orders of magnitude smaller than the energies that prevailed near the 'bang'. No Earth-based particle accelerator can possibly recreate such dramatic conditions. What has been done since the early 1980s is to extrapolate our theoretical models of particle physics to the extreme conditions of the very early universe, hoping to find possible clues of what went on. Countless scenarios have been proposed, suggesting the existence of new symmetries such as 'supersymmetry', and of 'topological defects', such as cosmic strings, tubes of energy that lock extreme high-energy conditions in their interior, possible relics of earlier times when the universe was more symmetric than today.

Supersymmetric theories are a continuation of Einstein's dream of unification, as they propose a complete joining of the four (known) forces of Nature into a single theory known as string theory. Not to be confused with the cosmic strings, such fundamental strings represent a deep ontological shift in the fabric of matter, as they would become the primal stuff of material existence: everything else, that is, all elementary particles of Nature, would be different vibrations of the fundamental strings, as different notes of a violin are different vibrations of its strings. In the 1980s, such theories got a tremendous boost, as they promised to unify all of Nature's building blocks - matter and forces - into a single scheme 
with only one free parameter. If such theory were to exist it would be a true Keplerian-Einsteinian marvel, the ultimate Platonist triumph, a Universe of Being, steeped in symmetry and simplicity.

Alas, during the past three decades that initial furore has been continuously pounded by the technical difficulties of the theory and by a series of results that indicate that the dream of a single string solution was not to be; indeed, currently it is proposed that there are over 10500 of such solutions, each representing a potential universe, each of these universes with different fundamental constants and thus physical properties. From being THE solution to a single string theory, our universe became just one among an outrageously large number of universes, none more essential or fundamental than the other: so much for our uniqueness. We could call this discovery, known as the string landscape, as the ultimate Copernican insult to our human dignity.

Furthermore, the expectation that supersymmetric particles predicted by various models would be discovered at the Large Hadron Collider has also been curbed by a distressing experimental silence. As of this writing, there is absolutely no evidence that such symmetry of Nature exists. If the results persist to the end of this decade, supersymmetry would have to be abandoned and, with it, so would superstrings. (It already has by many, although diehards argue that Nature could have opted for more complicated versions of the theory, unfortunately beyond current and future experimental reach, at least for many decades. One must wonder at what point do we declare a theory dead!).

Topological defects have not fared much better. They were supposedly created during a so-called symmetry-breaking phase transition in the early universe, not unlike the one we observe as water turns to ice in a cold freezer. We detect imperfections in the ice, and such could be deemed defects. The ones formed in particle theory are a bit more abstract, but the essence of the idea is the same (Gleiser 1998). Unfortunately, observational signals that such entities were created in the early stages of the cosmic expansion have also failed to materialise, leaving little room for their existence. They would be the Becoming alternative for extending our current theories to the dramatic conditions near the big bang. As it stands, we must drive in the dark, pushing our theories without much experimental guidance.

Hope emerged in the early 1980s, when Alan Guth proposed the inflationary scenario, whereby the early universe undergoes a short-lived ultra-fast period of expansion because of a mechanism inspired by symmetry-breaking. Guth's theory has been the greatest success in our current extrapolations, inspiring thousands of articles exploring its details and potential impact on the physics of the early and current Universe. According to inflationary cosmology, the universe's geometry should be effectively flat, in agreement with current observations. It also proposes a robust mechanism to generate the energy fluctuations that will later seed the growth of galaxies and their groups, an element missing in previous formulations of big bang models. It even revisits the definition of the big bang itself, now not the beginning of time, but the fiery end of the period of rapid expansion marked by explosive particle creation. Currently, we cannot say much about what happens before inflation without going deep into speculative physics. We also can't call inflation a theory, because we do not have the framework from particle physics to justify it. But we can call it an idea that seems to have the essential elements of a future successful theory.

Metaphysically, the inflationary universe deftly combines Being and Becoming in a rather unexpected way. The generation of rapidly growing energy fluctuations and the dramatic heating of the universe at the end of inflation have strong elements of becoming, of change and transformation. However, one of the unexpected consequences of the inflationary scenario, one that has received both praise and severe criticism (Ellis \& Silk 2014:321), is that it also predicts the existence of a multiverse, albeit one different from the string landscape discussed above. The essential mechanism driving inflation is the release of energy from a master field known as the 'inflaton'. Just as a child going down a slide releases gravitational potential energy, the inflaton releases its potential energy as it approaches its point of final rest at the lowest energy level allowed by its dynamics. However, contrary to a child going down a slide, the inflaton is susceptible to random quantum kicks, or fluctuations, that can drive it up or down as it evolves along its path. If we picture such phenomenon in space, we get a scenario where the universe resembles a blotchy painting, with different patches having the inflaton doing different things: in some, going up; in others, going down. Why is there a difference? Each region or patch is causally connected, that is, as big as light has had the time to travel in that short time interval. But the physics at different regions is not causally connected, and the inflaton could then evolve in very different ways. Each of these patches becomes a separate universe and, in different theories, with different physical properties. The random dynamics of the inflaton field creates a multiverse of possibilities that could extend to infinite space, at least in principle. Although each individual patch is rich in its own physics and dynamics, overall the multiverse just is a bubbling collection of cosmoids. It could exist forever, outside time, an entity that reminds us of Anaximander's Boundless, from whence everything came, to where everything returns.

Critics are quick to argue that the multiverse is not a true physical entity, as its existence cannot be empirically validated (Gleiser 2014): given that adjacent universes lie outside each others' causally connect realms, we could never directly infer the existence of another universe. This opens a complex precedent in theoretical physics, whereby an idea may inspire the development of models but cannot in itself be verified. If inflation prevails as a theory of the early universe, physicists will have to deal with this unknowable aspect of physical reality. Even so, the multiverse does not solve the First Cause, 
only shifts it to a deeper hierarchical level. For one can always ask why should there be a multiverse at all or where the inflaton comes from, questions no current theory is equipped to answer.

\section{Concluding remarks}

Starting with the metaphysical tension between Being and Becoming in creation myths across a diversity of cultures, we ended with an analysis of the same tension in current cosmological theories. It seems clear that both modes of thinking contribute to the construction of cosmic narratives, mythic and scientific. We identified several archetypical constructions that are replicated in mythic and scientific models, emphasising the key difference that, in the case of science, we can narrow the field by empirical observations. We are thus left with the most fundamental unknowable of all cosmic questions, the problem of the First Cause, or why there is something rather than nothing. We have argued that science is not equipped conceptually to address such question and, indeed, possibly neither is the human mind. We must live with our ignorance, surrounded by the mystery of existence, trying hard to expand the shores of our island of knowledge.

\section{Acknowledgements}

The author would like to thank Prof. Cornelius Du Toit for his invitation and generous hosting during this event.

\section{Competing interests}

The author declares that she has no financial or personal relationships which may have inappropriately influenced her in writing this article.

\section{References}

Alpher, R. \& Herman, R., 2001, Genesis of the big bang, Oxford University Press, Oxford, UK.

Aristotle, Metaphysics, Part X, viewed n.d., from http://classics.mit.edu/Aristotle/ metaphysics.12.xii.html on July 20th, 2017

Barrow, J. \& Tipler, F., 1988, The anthropic cosmological principle, rev. edn., Oxford University Press, Oxford, UK.

Copernicus, N., 1543, On the revolutions of the heavenly spheres, viewed n.d., from http://www.geo.utexas.edu/courses/302d/Fall_2011/Full\%20text \%20-\%20 Nicholas \%20Copernicus, \%20_De\%20Revolutionibus $\% 20 \% 280$ n $\% 20$ the $\% 20$ Revolutions\%29,_\%201.pdf

Einstein, A., 1952, The principle of relativity, Dover, New York.

Ellis, G. \& Silk, J., 2014, 'Defend the integrity of physics', Nature 516, 321-323. https:// doi.org/10.1038/516321a

Gleiser, M., 1997, The dancing universe: From creation myths to the big bang, Dutton, New York.

Gleiser, M., 1998, 'Phase transitions in the Universe', Contemporary Physics 39(4), 239-253. https://doi.org/10.1080/001075198181937

Gleiser, M., 2014, The Island of knowledge: The limits of science and the search for meaning, Basic Books, New York.

Hawking, S. \& Ellis, G., 1973, The large-scale structure of space-time, Cambridge University Press, Cambridge, UK.

Heraclitus, Fragments (c. 500 BCE), viewed 20 July 2017, from http://www. heraclitusfragments.com/Fragments.html

Kirk, G.S., Raven, J.E. \& Schofield, M. 1983, The pre-socratic philosophers: A critical history with a selection of texts, 2 nd edn., Cambridge University Press, Cambridge, UK.

Koyré, A., 1973, The astronomical revolution: Copernicus-Kepler-Borelli, Dover, New York.

Lanczos, C., 1970, The variational principles of mechanics, 4th edn., University of Toronto Press, Toronto.

Nagel, T., 1986, The view from nowhere, Oxford University Press, Oxford.

Newton, I., 1687, Mathematical principles of natural philosophy, transl. I. Bernard Cohen and A. Whitman, University of California Press, San Francisco, CA, Tra edition 2016.

Penrose, R., 1965, 'Gravitational collapse and space-time singularities', Physical Review Letters 14, 57. https://doi.org/10.1103/PhysRevLett.14.57

Plato, The republic, Book VII (360 BCE), viewed 20 July 2017, from http://classics.mit. edu/Plato/republic.mb.txt

Sproul, B., 1991, Primal myths: Creation myths around the world, HarperCollins, New York.

Westfall, R., 1983, Never at rest, A biography of Isaac Newton, Cambridge University Press, Cambridge, UK.

Weyl, H., 1922, Space, time, matter, Dover, New York. 